\title{
A Symbolic Simulator Platform for the Static Analysis of Thin Plates
}

http://dx.doi.org/10.3991/ijoe.v12i04.4963

\author{
D.M.S. Costa $^{1}$, G.M.S. Bernardo ${ }^{1,2}$ and M.A.R. Loja ${ }^{1,2}$ \\ ${ }^{1}$ Instituto Superior de Engenharia de Lisboa, Portugal \\ ${ }^{2}$ Universidade de Lisboa, Portugal
}

\begin{abstract}
The integration of symbolic capabilities into a simulation platform devoted to the analysis of thin plates can provide a greater flexibility in the learning process as it enables to incorporate, to experiment and simulate more complex and user-defined characteristics. With the present work one has built a symbolic simulator for the study of the mechanical behaviour of thin plates. These plates, with a rectangular/square basis geometric configuration, are simply supported along its four edges, and can be made from homogeneous or non-homogeneous materials. The capabilities of this platform are briefly illustrated through an illustrative case study.
\end{abstract}

Index Terms-Homogeneous and non-homogeneous materials, symbolic simulation platform, linear static analysis, thin plates.

\section{INTRODUCTION}

The static behaviour of plates is an important issue within the structural engineering field, which possesses transversal competences in Aerospace, Mechanical and Civil Engineering, among others. Thus the availability of tools that enable the analysis of such type of structures without a significant computational cost is an important matter, not only because in some situations there is no need to use more powerful applications, for instance finite element applications, but also because if we are talking about a B.Sc. student, their knowledge about this numerical technique may be more superficial. This simulator can also be very useful for M.Sc. students, as it enables the inter-connection of competences between the first cycle and the second cycle educations. The extension of the static analysis to non-isotropic and non-homogeneous materials and the link to gradient-based optimization concepts are also other capabilities present. With the present simulator, developed in the symbolic computation environment Maple (C) it is possible to perform static analyses according to the classical bending plate's theory assumptions, thus helping to improve broadening the scope of the student's knowledge.

\section{NAVIER METHOD}

The static response of a thin plate, undergoing small displacements, is ruled by the differential equilibrium equation (1):

$$
\frac{\partial^{4} w}{\partial x^{4}}+\frac{\partial^{4} w}{\partial y^{4}}+2 \frac{\partial^{4} w}{\partial x^{2} \partial y^{2}}=-\frac{p(x, y)}{D}
$$

submitted to the boundary conditions in (2), (3)

$$
\begin{aligned}
& w=0, \frac{\partial^{2} w}{\partial x^{2}}=0 \quad \text { when } x=0, x=a \\
& w=0, \frac{\partial^{2} w}{\partial y^{2}}=0 \quad \text { when } y=0, y=b
\end{aligned}
$$

One possible solution to this problem is based on the Navier method, where the transverse deflection is modelled through a double series Fourier series, as in (4):

$$
w(x, y)=\sum_{m=1}^{M} \sum_{n=1}^{N} W_{m n} \sin \left(\frac{m \pi x}{a}\right) \sin \left(\frac{n \pi y}{b}\right)
$$

and the load is also modelled in a similar way (5):

$$
p(x, y)=\sum_{m=1}^{M} \sum_{n=1}^{N} P_{m n} \sin \left(\frac{m \pi x}{a}\right) \sin \left(\frac{n \pi y}{b}\right)
$$

The coefficients $W_{m n}$ and $P_{m n}$ are given in (6) and (7):

$$
\begin{aligned}
P_{m n}= & \frac{4}{a b} \int_{0}^{a} \int_{0}^{b} \bar{p}(x, y) \sin \left(\frac{m \pi x}{a}\right) \sin \left(\frac{n \pi y}{b}\right) d x d y \\
W_{m n} & =\frac{P_{m n}}{\pi^{4} D\left[\left(\frac{m}{a}\right)^{2}+\left(\frac{n}{b}\right)^{2}\right]^{2}}
\end{aligned}
$$

As one can observe, the modelling approximations associated to the load and transverse deflection, depend on the number of terms in the double series expansion, denoted by $m$ and $n$, among other parameters, namely the elastic stiffness of the plate $D$ and the applied load $\bar{p}(x, y)$. The elastic stiffness of the plate (8) depends not only on geometrical but also on material characteristics of the plate. For the simplest case of a homogeneous isotropic material this parameter is given as:

$$
D=\frac{E h^{3}}{12(1-v)^{2}}
$$

where $E, v$ represent respectively the Young's modulus and Poisson's ratio and $h$ is the thickness of the plate.

\section{FUNCTIONALLY GRADED MATERIAL}

Being possible to consider different type of materials, we will use in the present paper, particulate composites 
known as functionally graded materials (FGM). These materials have been object of a great research effort related to the development and application of new materials in structural engineering, being possible to find them on the composition of several aerospatial structures, as space planes bodies or rocket engine components [1]. Also in other fields such as Defense, FGM have a particular ability to inhibit crack propagation, which can be very useful for armour plates and bullet-proof vests [2], due to its continuously varying in space, properties. But also in quiet different scientific areas such as the biomedical and bioengineering, we can find them, due to the fact that living tissues can be seen as natural FGM's, being the reason why these graded materials are ideal candidates to replace natural tissues in applications such as teeth and bones replacement [3].

In the present paper a case study devoted to the static analysis of a dual-phase FGM plate, and its main steps using the developed didactical simulator will be illustrated through a set of interactive windows. An aspect that it is also worth to note, as it eases and guides the use of this application, is the friendly interface to the user.

\section{CASE STUDY}

The overall procedure and usage/interaction scheme to the simulator is here briefly described. The case that we are about to study is a rectangular plate with all its four edges simply supported. This plate will be made from an advanced particulate composite, which is a globally nonhomogeneous material. In order to be able to proceed with the intended analysis, we will consider a homogenization procedure in order to obtain the associated equivalent average material properties. After this phase, and having defined the load applied to the plate, we will characterize its transverse deflection profile as well as the subsequent generalized resultant forces and stresses.

\section{A. Plate Geometry}

The definition of the case that is going to be considered starts by the characterization of the plate geometry data, the material that constitutes it and the load that acts transversely to the plate plane. To this purpose, one starts to select the Definition of the Plate's Geometry at the main window panel. The edges' dimensions input as well as the thickness of the plate, is done through the window that is presented in Fig. 1.

The graphical representation of the plate is also obtained according to the geometrical characteristics introduced.

\section{B. Material definition}

The plate can be made from materials corresponding to three different constitutive relations, namely concerning to isotropic, orthotropic and functionally graded materials. This selection is made through the Definition of Plate's Material button at the main panel, which leads to the window shown in Fig. 2.

In the present work, one has considered that the plate would be made from a dual-phase functionally graded material, constituted by silicon-carbide and titanium. In this situation it is now necessary to proceed to a homogenization step, which is carried out by selecting the Homogenization of Material Properties button. The equivalent average material properties can be then estimated via Voigt or Mori-Tanaka homogenization schemes, as seen

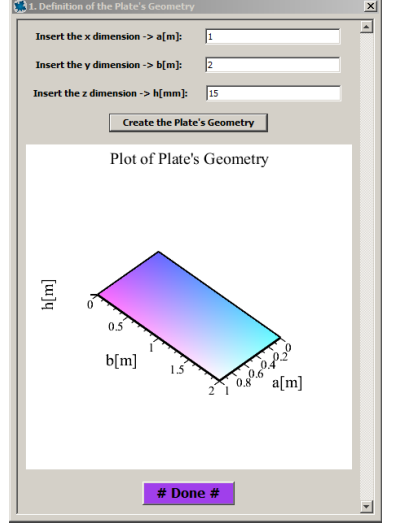

Figure 1. Definition of plate geometric characteristics.

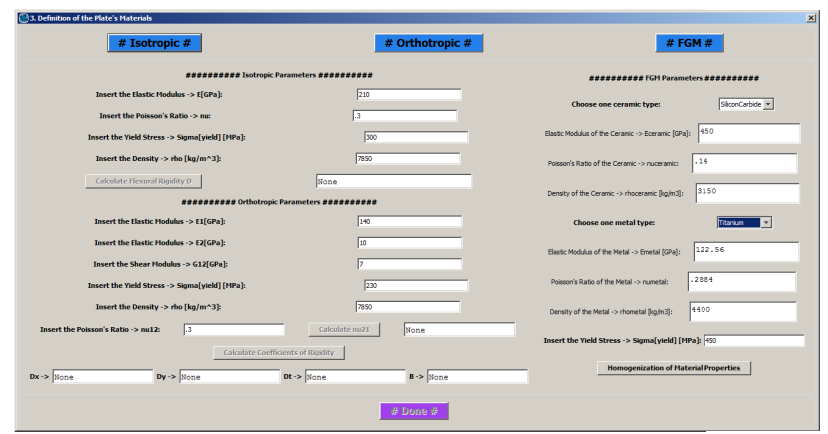

Figure 2. Selection of Material.

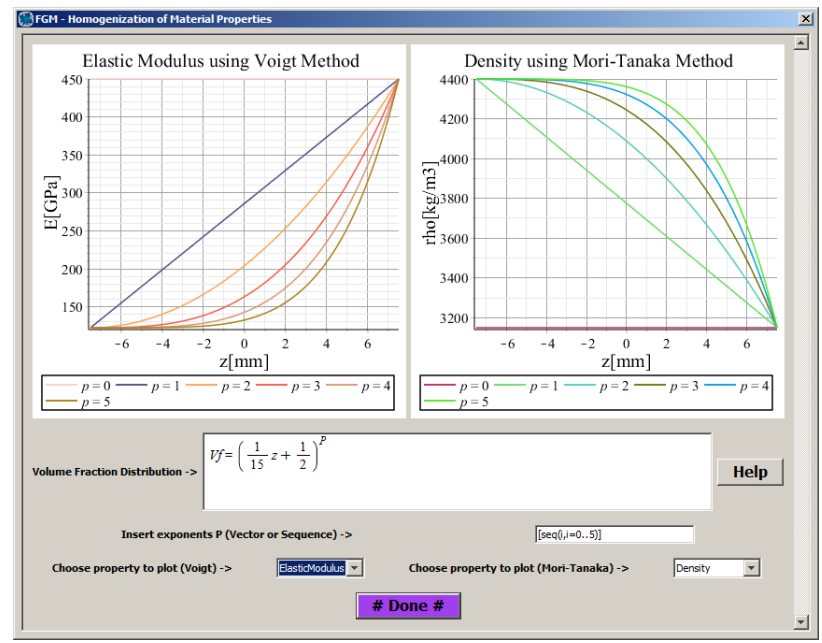

Figure 3. FGM - Homogenization of Material Properties.

in Fig. 3. The influence of using these approaches among others was studied by several researchers, as reported in [4]. Here it is possible to select not only the law that dictates the volume fraction distribution $V f$, through the thickness, but also to visualize the properties throughthickness, for user-defined exponents in the context of power-law distributions.

The values of this exponent $P$ enable a sooner or later faster inclusion of the reinforcement silicon carbide particles in the titanium matrix, thus influencing the properties distribution of the composite and the corresponding averages. The properties available in the platform are the elasticity modulus, the density and the Poisson's ratio. With the geometric and material properties defined, we need finally to define the load applied to the plate, in order to proceed to the static analysis of the plate. 


\section{Load Modelling}

Being possible to select a few typical cases of loads that appear often in everyday applications, it is however possible to define a desired load distribution. To this purpose, one needs to select Definition of Loads at the main panel. The window that will enable this definition is shown in Fig. 4.

The load selected in this paper corresponds to a distributed load in a rectangular sub-domain of the plate, which consists on the third option pre-defined, available at the Choose Load field at the window left side. The maximum value of the distributed load was set to $750 \mathrm{~Pa}$. In Fig. 4 it is also possible to see the load modelling using 20 terms in the Fourier expansion associated to each direction, and the 3D plot of the deviation distribution between two expansions using 1 and 20 terms, respectively. All these aspects are user-defined, easing his/her study and results' analysis.

\section{Transverse Deflection}

At this point, it is finally possible starting to achieve the characterization of the static behaviour of the plate. By selecting the Transverse Deflection option in the main panel, the user is lead to the window shown in Fig. 5.

In this figure we can observe the plate deflection profile for a volume fraction distribution using an exponent $P=0$, using $M=N=10$ terms and considering the Voigt homogenization scheme. Other quantities of interest, namely deviations, convergence trend of maximum deflection values, maximum values for deflection deviations along with the corresponding locations are also obtained. In the present case a $2 \mathrm{D}$ contour graph was used to visualize the deviations' isolines, but it is also possible to obtain the corresponding $3 \mathrm{D}$ representation.

\section{E. Resultant Generalized Forces and Stresses}

Associated to the transverse deflection profile obtained for this case study, we can now proceed to a postprocessing phase wherein one obtains the resultant shear forces and moments as well as the stresses distributions. This feature is available at the main panel and leads to the window illustrated in Fig. 6, where it is additionally possible to get the maximum values associated to each distribution as well as the associated locations.

Considering that in these plates we have a bi-axial state of stress, it may be important to be able to characterize an equivalent stress measure, which in the present simulator is done by using the Von-Mises stress. It is also possible to determine the design safety factor. These quantities may be very useful if considering a plate design context.

\section{F. Concluding Remarks}

An educational simulator was developed to support plate's learning and teaching in structural, civil and mechanical engineering at B.Sc. and M.Sc. levels. The functionalities available allow for an easy perception of the multidisciplinary concepts involved in the static analysis of thin plates, easing and improving the process of competences acquisition and application.

\section{ACKNOWLEDGMENT}

The authors wish to acknowledge the financial support given by FCT/MEC through Project PTDC/ATPAQI/5355/2012 and Proj. LAETA-UID/EMS/50022/2013.

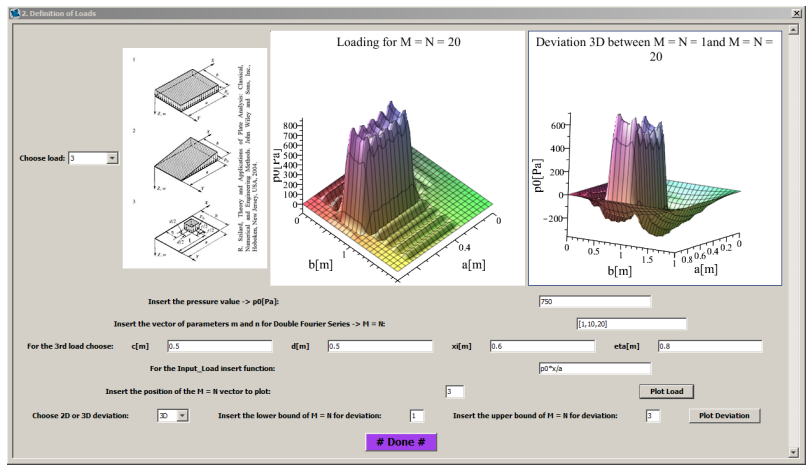

Figure 4. Load modeling and characterization.

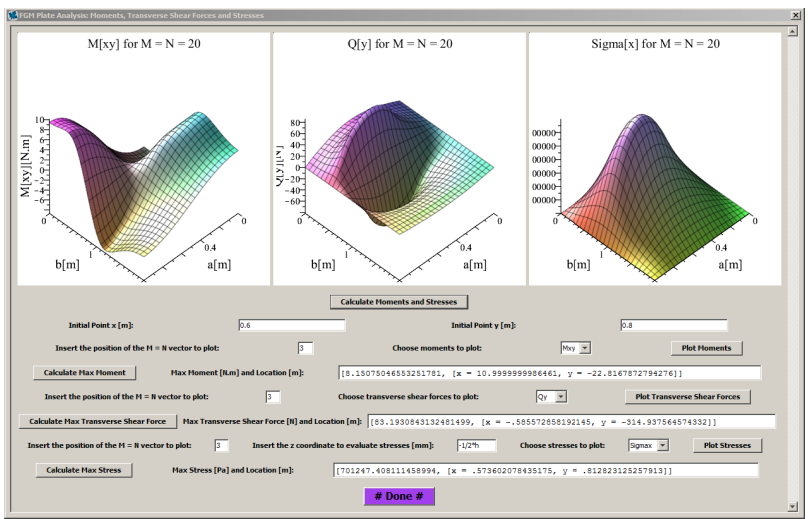

Figure 5. Resultant generalized forces and stresses on the FGM plate

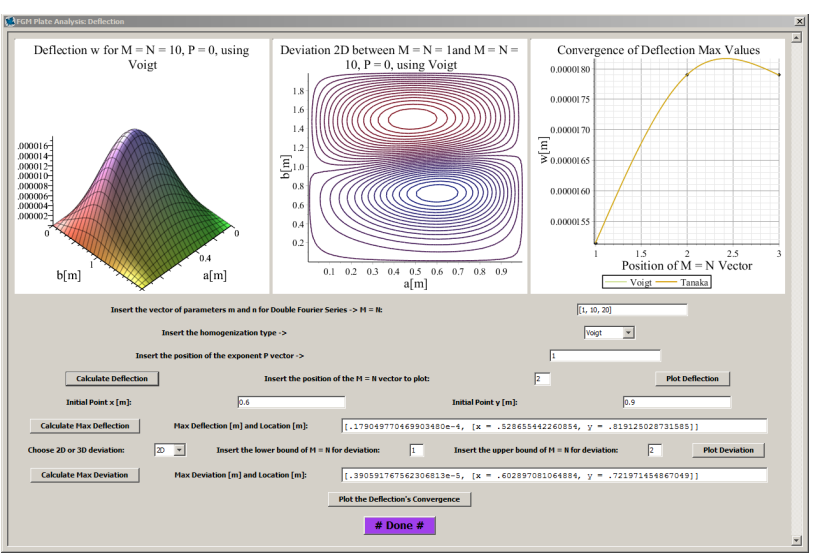

Figure 6. Transverse deflection analysis of FGM plate

\section{REFERENCES}

[1] L.Marin, "Numerical solution of Chauchy problem for steady-state heat transfer in two dimensional functionally graded materials", Int J Solids Struct, vol. 42, (2005), pp. 4338-4351 http://dx.doi.org/10.1016/j.ijsolstr.2005.01.005

[2] L. Lu et al, "Mechanical properties estimation of functionally graded materials using surface waves recorded with a laser interferometer", NDT \&amp; E International, vol. 44, Issue 2, (2011), pp. 169-177 $\quad$ http://dx.doi.org/10.1016/j.ndteint. 2010.11 .007

[3] F. Watari et al, "Biocompatibility of materials and development to functionally graded implant for bio-medical application", Compos Sci Tecnhol, vol. 64, (2004), pp. 893-908. http://dx.doi.org/10.1016/j.compscitech.2003.09.005

[4] M.A.R. Loja, J. I. Barbosa, C. M. Mota Soares, "A Study on the Modeling of Sandwich Functionally Graded Particulate Composites", Composite Structures, vol. 94(7) 2209-2217, 2012. http://dx.doi.org/10.1016/j.compstruct.2012.02.015 
PAPER

A Symbolic Simulator Platform for the Static ANAlysis of Thin Plates

\section{AUTHORS}

D.M.S. Costa is with GI-MOSM, Grupo de Investigação em Modelação e Optimização de Sistemas Multifuncionais and ISEL, IPL - Instituto Superior de Engenharia de Lisboa, Portugal (a38293@alunos.isel.pt)

G.M.S. Bernardo is with GI-MOSM, Grupo de Investigação em Modelação e Optimização de Sistemas Multifuncionais and ISEL, IPL - Instituto Superior de Engenharia de Lisboa, Portugal, and LAETA, IDMEC, Instituto
Superior Técnico, Universidade de Lisboa, Portugal (goncalo.bernardo@tecnico.ulisboa.pt)

M.A.R. Loja Bernardo is with GI-MOSM, Grupo de Investigação em Modelação e Optimização de Sistemas Multifuncionais and ISEL, IPL - Instituto Superior de Engenharia de Lisboa, Portugal, and LAETA, IDMEC, Instituto Superior Técnico, Universidade de Lisboa, Portugal (amelialoja@dem.isel.ipl.pt)

Submitted 11 August 2016. Published as resubmitted by the authors 23 November 2015. 